\title{
Manejo da sede: perspectiva do paciente cirúrgico queimado
}

\section{Thirst management: perspective of burnt surgical patient}

\author{
Renata Pires de Arruda Faggion ${ }^{1}$, Roberto Emanuel Bueno \\ Ferreira $^{2}$, Aline Korki Arrabal Garcia ${ }^{3}$, Isadora Pierotti ${ }^{4}$, \\ Marilia Ferrari Conchon ${ }^{5}$, Lígia Fahl Fonseca ${ }^{6}$
}

Resumo

\begin{abstract}
Objetivo: explorar a percepção de um paciente cirúrgico queimado em relação à sede e seu manejo no período pré-operatório e pós-operatório imediato. Relato de caso: trata-se de um estudo com abordagem qualitativa, exploratória, do tipo estudo de caso. Os critérios de inclusão foram: paciente estar internado no centro de tratamento de queimados, ser submetido a procedimento cirúrgico ou balneoterapia, ter experenciado a sede no período pré-operatório ou pós-operatório e ter recebido o manejo da sede. Para a coleta de dados utilizou-se entrevista semiestruturada, gravada e transcrita. Paciente do sexo feminino, de 32 anos, admitida com queimaduras de segundo grau em extensão de tórax, membros superiores e pescoço por tentativa de autoextermínio com álcool. Passou por seis procedimentos e esteve internada por 15 dias até o momento da coleta. Experienciou o desconforto sede durante o jejum pré-operatório e pós-operatório, considerado intenso e muito estressante durante sua internação. Conclusão: a partir da identificação do desconforto sede, utilizou-se como estratégia o picolé de gelo, que fez diferença em seu tratamento. O modelo de manejo da sede é pioneiro no cuidado ao paciente queimado e apresenta benefícios para minorar a sede.
\end{abstract}

Palavras-chave: Sede; Queimaduras; Enfermagem perioperatória; Unidades de queimados; Xerostomia.

\begin{abstract}
Objective: explore thirst perception of a burnt surgical patient and its management in the preoperative and immediate postoperative period. Case report: study with a qualitative and exploratory approach, named as case study. Inclusion criteria were: inpatient at burnt treatment unit, undergone surgical procedure or balneotherapy, experienced thirst in the preoperative or postoperative period and received
\end{abstract}

${ }^{1}$ Graduanda em Enfermagem na Universidade Estadual de Londrina (UEL), Londrina, Paraná, Brasil. E-mail: renatafaion@ hotmail.com

${ }^{2}$ Graduando em Enfermagem na Universidade Estadual de Londrina, Londrina, Paraná, Brasil.

${ }^{3}$ Doutoranda no Programa de Pós-graduação em Enfermagem da Universidade Estadual de Londrina, Londrina, Paraná, Brasil.

${ }^{4}$ Doutoranda no Programa de Pós-graduação em Enfermagem da Universidade Estadual de Londrina, Londrina, Paraná, Brasil. Professora Temporária no Departamento de Enfermagem do Centro de Ciências da Saúde (CCS) da Universidade Estadual de Londrina, Londrina, Paraná, Brasil.

${ }^{5}$ Doutorado em Ciências pelo Programa de Pós-graduação em Enfermagem Fundamental da Escola de Enfermagem de Ribeirão Preto da Universidade de São Paulo (EERP-USP), Ribeirão Preto, São Paulo, Brasil. Professora do Departamento de Enfermagem do Centro de Ciências da Saúde da Universidade Estadual de Londrina, Londrina, Paraná, Brasil.

${ }^{6}$ Doutorado em Enfermagem pelo Programa de Pós-graduação em Enfermagem na Saúde do Adulto da Escola de Enfermagem da Universidade de São Paulo (EEUSP), São Paulo, São Paulo, Brasil. Professora Associada do Departamento de Enfermagem da Universidade Estadual de Londrina, Londrina, Paraná, Brasil. 
thirst management. For data collection, semi-structured interviews were performed, recorded and transcribed. Female patient, 32 years old, admitted with second degree burns in extension of thorax, upper limbs and neck due to attempted self-extermination with alcohol. Underwent six procedures and was hospitalized for 15 days until the data collection. Experienced thirst discomfort during preoperative and postoperative fasting, which was considered intense and very stressful during his hospitalization. Conclusion: since the identification of thirst discomfort, the ice popsicle was used as a strategy, which made difference in her treatment. The thirst management model is pioneer in care of burnt patients and has benefits to alleviate thirst.

Keywords: Thirst; Burns; Perioperative nursing; Burn units; Xerostomia.

\section{Introdução}

O período perioperatório é considerado um momento angustiante e de incertezas para o paciente, ${ }^{(1)}$ e engloba inúmeros desconfortos, desde o preparo para a cirurgia até o pós-operatório. Desses incômodos, alguns são facilmente identificados e tratados pela equipe perioperatória, como a dor, as náuseas, os vômitos e a hipotermia. Observa-se, no entanto, que a sede também é um sintoma presente e intenso no paciente cirúrgico e pouco valorizado pela equipe de saúde. ${ }^{(2-3)}$

A sede perioperatória pode ocorrer por uma confluência de fatores. É deflagrada com a restrição hídrica durante o período de jejum pré-operatório, intensificando-se pelas medicações anestésicas e perdas sanguíneas e pela intubação orotraqueal no intra-operatório, e revelando sua face mais estressora durante o pós-operatório imediato (POI). ${ }^{(4-6)}$

A prevalência da sede no POI em Sala de Recuperação Anestésica (SRA) é de 89,6\% em pacientes adultos ${ }^{(7)}$ podendo superar até mesmo a dor e a fome. ${ }^{(8-9)}$ Esse desconforto é caracterizado por sinais, como boca e garganta secas, lábios ressecados, língua e saliva grossas, gosto ruim na boca e vontade de beber água. ${ }^{(10)}$ Além disso, alterações emocionais podem advir da sede intensa; dentre elas podemos destacar a ansiedade, a irritabilidade e o desespero. ${ }^{(4)}$

Embora esses dados sejam referentes ao paciente cirúrgico em $\mathrm{POI}$, observa-se na prática clínica que a sede também é prevalente e intensa no paciente cirúrgico queimado. Isso ocorre devido à fisiopatologia da queimadura, que poderá acarretar hipovolemia, ${ }^{(11-12)}$ necessidade de uso diário de opioides para controle da dor, ${ }^{(13-14)}$ e sobretudo devido aos frequentes períodos de jejum prolongado para a realização de múltiplos procedimentos cirúrgicos e balneoterapias. ${ }^{(15)}$

Diante da restrição hídrica recorrente imposta ao paciente cirúrgico queimado, foi implantado em um Centro de Tratamento de Queimados (CTQ) o Modelo de Manejo da Sede Pré-operatória. ${ }^{(6)}$ Este modelo tem como objetivos identificar, mensurar e avaliar a segurança para o manejo e ofertar uma estratégia de alívio ao paciente queimado durante o período de jejum pré-anestésico.

Neste cenário, este relato de caso teve como objetivo explorar a percepção de um paciente cirúrgico queimado em relação à sede e seu manejo no período pré-operatório e pós-operatório imediato.

\section{Método}

Trata-se de um estudo com abordagem qualitativa, exploratória, do tipo relato de caso, o qual propõe uma investigação aprofundada com o intuito de compreender uma realidade. ${ }^{(16)}$

A pesquisa foi realizada em um hospital universitário de grande porte do Sul do Brasil, centro de referência para trauma, tratamento de queimados, transplante de medula óssea e gestação de alto risco. É uma entidade pública sem fins lucrativos, que atende exclusivamente o Sistema Único de Saúde (SUS) e possui 315 leitos.

O CTQ do hospital atende todas as faixas etárias, com disponibilidade de 10 leitos de enfermaria e seis leitos de Unidade de Terapia Intensiva (UTI). Além disso, conta com duas salas cirúrgicas e uma sala para balneoterapias. 
Os critérios de inclusão para a pesquisa foram: paciente estar internado no CTQ, ser submetido a procedimento cirúrgico ou balneoterapia, ter experenciado a sede no período pré-operatório ou pós-operatório imediato e ter recebido o manejo da sede.

Para a coleta de dados utilizou-se entrevista semiestruturada, gravada e transcrita contemplando as seguintes questões norteadoras: "qual a explicação que recebeu sobre o jejum?”; "o que sentiu ao permanecer em jejum?"; "você verbalizou a sua sede para algum profissional?"; "o que disseram?”; "quais os desconfortos que sentiu com relação à sede?"; "qual foi sua sensação ao receber o picolé de gelo?”; “o que isso representou para você?”; e "conte-me mais sobre isso".

A pesquisa faz parte de um projeto maior, denominado "Implantação do modelo de manejo da sede perioperatória: estudo de intervenção norteado pelo knowledge translation", aprovado pelo comitê de ética da instituição pesquisada (CAAE: 13638519.1.0000.5231). A participante foi questionada sobre sua disponibilidade em participar da pesquisa e informada como seria sua participação. Devido à queimadura ter atingido os membros superiores, encontrava-se impossibilitada de assinar o Termo de Consentimento Livre e Esclarecido (TCLE) de próprio punho, o qual foi assinado por sua irmã.

\section{Relato de caso}

Paciente do sexo feminino, de 32 anos, residente na cidade de Guaíra, no interior do estado do Paraná, Brasil. Admitida em um hospital universitário no dia 27 de fevereiro de 2020, com queimaduras de segundo grau localizadas em região de extensão de tórax, membros superiores e pescoço, totalizando $15 \%$ da superfície corporal queimada (SCQ) por tentativa de autoextermínio com álcool. Realizou seis procedimentos até o momento da coleta de dados, entre eles balneoterapias, traqueostomia, desbridamentos e enxertos. Como parte da terapêutica recebeu analgesia, hidratação por via endovenosa e antibióticos.
De início, a paciente foi internada na UTI do CTQ por um período de nove dias, onde necessitou de ventilação mecânica, sendo inicialmente realizada intubação orotraqueal, e posteriormente traqueostomia.

Após a melhora do quadro clínico, foi encaminhada para a enfermaria do CTQ onde novamente necessitou do jejum pré-anestésico para a realização de outros procedimentos.

Quando questionada sobre as informações que recebia da equipe sobre o jejum pré-anestésico, a mesma relatou que os profissionais diziam ser necessário o período de jejum "por conta da anestesia". No entanto, "não ofertavam maiores explicações". Associou o tempo que ficou em jejum com momentos de sofrimento:

[...] sim, é sofrido, judia da gente e aqui nessa cama uma coisinha pequena faz a diferença.

Conforme relato da paciente, o horário de jejum se delongava devido aos atrasos dos procedimentos de outros pacientes, como ilustrado na fala a seguir:

[...] não tem um horário. Igual, às vezes eles falam que será de manhã. Igual ontem, falaram que a minha cirurgia seria de manhã, quando eu acordei, me arrumaram e só fui fazer às 14 horas, e eu nunca fiz no horário certo.

E mesmo experenciando sede, a entrevistada não falava espontaneamente para os profissionais e os mesmos não perguntavam. Contudo, uma enfermeira orientou a paciente que líquidos claros poderiam ser ingeridos até duas horas antes da cirurgia.

Diante disso, ela expressou uma afeição de felicidade, de alívio, pois finalmente poderia beber água, e se saciar:

Depois que eu descobri com a enfermeira que era duas horas antes... eu já fiquei das 10 horas até a meia-noite, até no outro dia, sem beber água, sem nada.

Ao ser questionada como se sentiu ao estar em jejum e a respeito da sede vivenciada durante o 
jejum pré-operatório e pós-operatório imediato, relatou que esse desconforto era frequente e intenso. Esclareceu que tem o hábito de ingerir uma grande quantidade de líquidos durante o dia e, portanto, a restrição de líquidos foi um momento considerado angustiante.

Eu tomo muita água, para mim a maior dificuldade nem é ficar com fome aqui. A minha maior dificuldade aqui é ficar sem tomar água. Minha boca fica seca, língua grossa meio marrenta assim, sabe, saliva, bastante saliva grossa assim, sabe, secura na garganta, a gente não consegue nem falar, nariz seco... (pausa).

Devido à intensa sede experenciada pela paciente, a mesma procurava estratégias para minorar a sede por conta própria.

[...] é a pior coisa do mundo, para mim é a pior coisa. Eu estou com a garrafinha de água toda hora molhando a boca. É horrivel, fica com uma língua grossa, garganta seca, nariz seco de não conseguir nem sair voz.

Por ter estado internada e intubada, que é um fator agravante para a xerostomia, por vários dias e passado por vários procedimentos, sua sede foi intensa. Outro fator que pode ter intensificado esse sintoma foi o uso de medicações, como opioides e anticolinérgicos. ${ }^{(8)}$

Vixe! É muito importante... Você quer ver quando eu estava intubada, o primeiro dia que tiraram eu do 'intubamento' e eu fui tomar água. Minha irmã tava junto comigo... Eu ficava: 'nega, fala para eles que eu preciso de água, água, eu preciso de água, pelo amor de Deus'. Desse jeito. Dai foi passando as horas e eles me deram água na seringa, sempre aos poucos. Até eu me acostumar.

O modelo de manejo da sede implantado no CTQ envolve identificação, mensuração, avaliação da segurança e a utilização de estratégias frias para aliviar a sede, dentre elas o picolé de gelo. Ao ficar ciente que poderia ingerir o picolé de gelo, imediatamente solicitou à equipe de enfermagem. Quando questionada quanto à sua experiência em ter recebido as quatro etapas do manejo da sede aplicada pela equipe de enfermagem, culminando com o picolé de gelo, a paciente falou a relevância que isso teve na sua experiência e vida durante a internação.

[...] foi muito bom o picolé, alivia bastante a boca. Essa secura que fica... alivia. Todo tempo minha boca tá seca, eu acabei de tomar água e ainda está seca, mas quando você coloca o picolezinho parece que alivia tudo, de verdade mesmo! É muito bom!

\section{Discussão}

O presente estudo é inovador ao abordar um tema relevante como a sede do paciente cirúrgico queimado, ainda inexplorado na literatura. Durante o relato da participante, foi possível observar como receber o manejo da sede, incluindo estratégias de alívio como o picolé de gelo ou poder ingerir água duas horas antes do procedimento, fez a diferença em seu tratamento.

A finalidade da restrição hídrica, e consequentemente o jejum pré-operatório, é garantir o esvaziamento gástrico, evitando assim a broncoaspiração associada à diminuição dos reflexos protetores do esfíncter esofágico ocasionada pela indução anestésica. ${ }^{(17-18)}$ No entanto, a American Society of Anesthesiologists (ASA) recomenda até duas horas de jejum para líquidos claros sem resíduos, seis horas para refeições leves e oito horas para refeições gordurosas como procedimentos seguros para evitar a broncoaspiração. ${ }^{(19)}$ Porém, o período de jejum pré-anestésico acima do recomendado ainda é frequentemente encontrado na prática clínica. ${ }^{(20)}$

O jejum prolongado somado ao trauma cirúrgico pode gerar estresse metabólico ao paciente. Isso ocorre devido a um processo fisiológico que passa por modificações catabólicas, aumentando o cortisol e o glucagon e diminuindo a insulina no organismo e, como consequência, ocorrendo processo inflamatório e resistência insulínica. Essas mudanças acarretam o prolongamento da hospitalização e os desconfortos da sede e da fome. ${ }^{(17-18,21)}$ 
No paciente queimado este cenário se agrava, pois pode apresentar um estado hipercatabólico, promovendo eventos como a gliconeogênese e a proteólise. Devido a sucessivas exposições ao jejum pré-anestésico, seu estado de estresse pode aumentar e comprometer as funções vitais e a cicatrização das feridas, por exemplo. ${ }^{(15)}$

Diante disso, a abreviação do jejum torna-se uma das estratégias para aliviar o estresse cirúrgico e a sede. No pré-operatório, bebidas com carboidratos podem ser administradas a fim de diminuir a resistência à insulina e para alívio da sede. . $22-23)^{2}$

Analisando-se a sede de acordo com a Teoria do Manejo dos Sintomas, a percepção do paciente que vivencia esse sintoma é relevante e o primeiro passo para a compreensão do impacto em sua experiência. A sede é um desconforto experimentado individualmente e possui relação com aspectos ambientais, emocionais e fisiológicos. ${ }^{(5,24-25)}$ A paciente deste estudo relatou hábito de grande quantidade de ingesta líquida antes de sua internação. Uma pesquisa realizada nos Estados Unidos da América indicou alterações nas concentrações plasmáticas de Arginina-Vasopressina (AVP) conforme o hábito de ingesta hídrica. Sendo assim, indivíduos que possuem o hábito de ingerir baixa quantidade de líquidos não apresentam manifestações fisiológicas relacionadas à sede, apesar de terem aumento nas quantidades plasmáticas de AVP. Entretanto, pessoas que têm o hábito de ingerir grande quantidade hídrica diariamente, quando privados de água podem vivenciar a sede mais intensamente. Isso pode ocorrer mesmo quando as concentrações plasmáticas de AVP estiverem baixas. Uma das hipóteses é que esse fator está ligado com as respostas diferentes dos indivíduos durante a detecção dos estímulos orofaríngeos. ${ }^{(26)}$

Considerando que o paciente cirúrgico queimado tem grande chance de experienciar sede intensa, é necessário que ocorra uma abordagem intencional do sintoma sede. Visando minorar esse desconforto, o Modelo de Manejo da Sede Préoperatória foi implantado no CTQ. ${ }^{(6)}$

Estudos do Grupo de Estudo e Pesquisa da Sede (GPS), o qual é vinculado ao departamento de enfermagem de uma universidade pública no estado do Paraná, identificaram uma subvalorização por parte da equipe em relação à sede: anestesiologistas, cirurgiões e equipe de enfermagem ${ }^{(2-4)}$ consideram a sede como um desconforto de menor impacto. Todavia, estudos qualitativos realizados com crianças e adultos indistintamente demonstram quão distressante é para o paciente cirúrgico suportar a sede por tempo prolongado, tanto no pré-operatório quanto no pós-operatório. ${ }^{(1,27)}$ A não verbalização espontânea do paciente com sede pode estar associada com a afirmação constante da equipe sobre o jejum pré-operatório, para que a cirurgia seja segura, e por acreditar que seja papel do profissional questionar sobre a sede. ${ }^{(28)}$

Neste relato de caso, evidencia-se o papel essencial da enfermeira que orientou a paciente sobre a possibilidade de ingestão hídrica até duas horas antes do procedimento anestésico e a existência do manejo da sede no CTQ. Uma vez que a equipe é essencial na reabilitação do paciente queimado, esta deve estar preparada para identificar situações estressantes para o paciente e minimizar o sofrimento durante sua hospitalização. ${ }^{(29)}$

Ademais, o cuidado requer o diálogo, a escuta, o ato de ouvir e de perguntar. ${ }^{(30)}$ Os profissionais devem perceber a importância de identificar a sede, desenvolvendo um diálogo de confiança com os pacientes a fim de melhor tratá-la. ${ }^{(5)} \mathrm{O}$ olhar com intencionalidade para o desconforto da sede, um incômodo despercebido pela maioria dos profissionais, ${ }^{(2-3)}$ traz à tona a necessidade da humanização do cuidado, proporcionando conforto e alívios físico e emocional. ${ }^{(30)}$

Estudos que avaliaram a percepção de anestesiologistas $^{(3)}$ e da equipe de enfermagem ${ }^{(2)}$ sobre a sede apontam que esse sintoma ainda é pouco percebido pela equipe no período perioperatório. Quando questionados sobre quais eram os principais desconfortos que percebiam nos pacientes, mencionaram dor, náusea e vômito, sem menção à sede. ${ }^{(2-3)}$ No entanto, em pacientes de terapia intensiva a sede foi ranqueada como o primeiro maior estressor, sendo desencadeado ou agravado por fatores como: doenças pré-existentes, fatores 
psicológicos, instabilidade hemodinâmica e ventilação mecânica, entre outros. ${ }^{(27)}$

Quando questionados sobre a sede não atendida, os pacientes dão voz ao intenso sofrimento que vivenciam durante este processo. Estudo qualitativo revelou que o paciente associa a sede intensa a pensamentos de morte e comparam a experiência ao trajeto de um camelo no deserto. ${ }^{(4)}$

A sede também pode ser percebida por sinais periféricos, como boca e garganta secas, lábios ressecados, saliva e língua grossas. ${ }^{(10)}$ No relato de caso, foi possível perceber que esses sinais foram vivenciados pela paciente.

Após receber o manejo da sede pela primeira vez, a paciente o solicitava para a equipe durante os períodos de jejum pré-anestésico e pós-anestésico seguintes. Essa escolha da paciente pode se dar devido ao fato de que o picolé de gelo é mais eficaz que a água em temperatura ambiente em minorar a sede. ${ }^{(31)}$ Sua atuação eficaz justifica-se pela ação da temperatura fria do picolé nos receptores orofaríngeos denominados Transient Receptor Potential Melastatin 8 (TRPM8), os quais, por meio de vias neurais, relacionam-se com áreas cerebrais específicas gerando a sensação de prazer e saciedade da sede. ${ }^{(32-35)}$

Durante a implantação do Modelo de Manejo da Sede Pré-operatória no CTQ foram estabelecidas as utilizações do picolé de gelo e do hidratante labial como estratégias de alívio do sintoma. O hidratante na recuperação da mucosa oral e na redução dos sinais periféricos (relatados pela paciente), como lábios ressecados. Este conjunto de ações fornecido pelos profissionais confere ao paciente alívio da sede, melhora a hidratação labial e diminui a secura e o gosto ruim na boca, proporcionando maior conforto e agradabilidade e assim facilita o percurso pelo qual irão enfrentar.

Diante das manifestações clínicas que acometeram a paciente, o sintoma sede foi o principal desconforto durante seu período de hospitalização. $\mathrm{O}$ sentimento em experenciar a sede trouxe à tona a angústia e o desespero devido à falta de água. A percepção do paciente cirúrgico queimado evidencia que vivenciar a sede é desagradável e ainda assim os sinais são subvalorizados pela equipe de saúde durante a assistência. Quando esse desconforto foi notado e o silêncio entre a paciente e a equipe foi quebrado, tornou-se possível ofertar estratégias para o manejo da sede, proporcionando alívio para sua maior dificuldade no momento.

\section{Conclusão}

A aplicação do Modelo de Manejo da Sede Pré-operatória é pioneira no cuidado ao paciente queimado. $\mathrm{O}$ fato de ser baseada em evidências e apresentar benefícios para minorar a sede ressalta a relevância de sua divulgação científica, a fim de que outros pacientes queimados possam ser contemplados com uma assistência mais humana e qualificada durante seu tratamento e ter sua percepção de sede minorada. Foi possível observar o benefício da aplicação do manejo da sede em reduzir o sofrimento da paciente, utilizando-se como estratégia o picolé de gelo, relatando que isso fez diferença em seu tratamento.

\section{Referências}

1 Camponogara S, Soares SGA, Silveira M, Viero CM, Barros CS, Cielo C. Percepções de pacientes sobre o período pré-operatório de cirurgia cardíaca. Rev Min Enferm. [Internet]. 2012. [citado 2021 mar 20];16(3):382-90. Disponível em: https://cdn.publisher.gn1.link/reme.org.br/ pdf/v16n3a10.pdf

2 Pavani MM, Fonseca LF, Conchon MF. Thirst in surgical patients: perceptions of the nursing team in inpatient units. Rev Enferm UFPE. 2016;10(9):3352-60. doi: 10.5205/reuo 1.957183638-1-SM1009201621.

3 Garcia ACKA, Nascimento LA, Conchon MF, Garcia AKA, Fonseca LF. Anesthesiologist's perspective regarding thirst in the immediate postoperative period. Ciên Cuid Saúde. 2017; 16(3). doi: 10.4025/cienccuidsaude.v16i3.37241.

4 Silva LCJR, Aroni P, Fonseca LF. I am thirsty! Experience of the surgical patient in the perioperative period. Rev SOBECC. 2016;21(2); 
75-81. doi: https://doi.org/10.5327/Z14144425201600020003

5 Conchon MF, Nascimento LA, Fonseca LF, Aroni P. Perioperative thirst: an analysis from the perspective of the Symptom Management Theory. Rev Esc Enferm USP. 2015;49(1):1228. doi: 10.1590/S0080-623420150000100016.

6 Nascimento LA, Garcia AKA, Conchon MF, Aroni P, Pierotti I, Martins PR, et al. Advances in the management of perioperative patients' thirst. AORN Journal. 2020;111(2):165-79. doi: https://doi.org/10.1002/aorn.12931.

7 Pierotti I, Fracarolli IFL, Fonseca LF, Aroni P. Avaliação da intensidade e desconforto da sede perioperatória. Esc Anna Nery. 2018;22 (3):e20170375. doi: 10.1590/2177-9465-ean2017-0375.

8 Arai S, Stotts N, Puntillo K. Thirst in critically III patients: from physiology to sensation. Am J Crit Care. 2013;22(4):328-35. doi: 10.4037/ ajcc2013533.

9 Gois CFL, Aguilar OM, Santos V, Rodríguez EOL. Stress factor for patients undergoing cardiac surgery. Invest Educ Enferm. [Internet]. 2012. [cited 2021 Abr 12];30(3):312-9. Available from: http://www.scielo.org.co/pdf/iee/ v30n3/v30n3a03.pdf

10 Martins PR, Fonseca LF, Rossetto EG, Mai LD. Developing and validating the perioperative thirst discomfort scale. Rev Esc Enferm USP. 2017;51:e03240. doi: 10.1590/S1980220X2016029003240.

11 Montanari T. Histologia: texto, atlas e roteiro de aulas práticas. $3^{\mathrm{a}}$ ed. Porto Alegre: UFRGS; 2016.

12 Singer AJ, Lee CC. Thermal burns. In: Walls RM, Hockberger RS, Gausche-Hill M, Bakes K, Baren JM, Erickson TB, et al. Rosen's emergency medicine concepts and clinical practice. $9^{\text {th }}$ ed. Philadelphia: Elsevier; 2018. p. 715-23.

13 Piccolo MTS, Daher RP, Daher SP, Alves Neto O. Dor em pacientes queimados. In: Alves Neto O, Costa CMC, Siqueira JTT, Teixeira MJ. Dor: princípios e prática. Porto Alegre: ARTMED; 2009. p. 847-57.
14 Castro RJA, Leal PC, Sakata RK. Tratamento da dor em queimados. Rev Bras Anestesiol. 2013;63(1):154-8. doi: 10.1590/S0034-70942 013000100013 .

15 Winckworth S, Allort NL, Clarke DL, Aldous C. Perioperative fasting in burn patients: Are we doing it right? S Afr J Surg. 2015;53(2): 65-6. doi: 10.7196/SAJSNEW.7743.

16 Yin RK. Estudo de caso: planejamento e método. $5^{\text {a }}$ ed. Porto Alegre: Bookman; 2015.

17 Silva AH, Miguez BB, Oliveira AP, Ferreira JM. A importância da redução do tempo de jejum pré-operatório: uma revisão literária. Rev Cad Med. [Internet]. 2019. [citado 2021 abr 10];2(2):174-82. Disponível em: http:// www.revista.unifeso.edu.br/index.php/ cadernosdemedicinaunifeso/article/ view/1389/604

18 Campos SBG, Barros-Neto JA, Guedes GS, Moura FA. Pre-operative fasting: why abbreviate? ABCD Arq Bras Cir Dig. 2018;31(2): e1377. doi: 10.1590/0102-672020180001e 1377.

19 American Society of Anesthesiologists. Practice guidelines for preoperative fasting and the use of pharmacologic agents to reduce the risk of pulmonary aspiration: application to healthy patients undergoing elective procedures: an updated report by the American Society of Anesthesiologists Task Force on Preoperative Fasting and the Use of Pharmacologic Agents to Reduce the Risk of Pulmonary Aspiration. Anesthesiology. 2017;126(3):376-93. doi: 10.1097/ALN.0000000000001452.

20 Nascimento LA, Fonseca LF, Santos CB. Inter-rater reliability testing of the safety protocol for thirst management. J Perianesth Nurs. 2016;33(3):527-36. doi: 10.1016/j.jopan.2016. 07.008 .

21 Marcarini M, Rosa SC, Wieck FP, Betti AH. Abreviação do jejum: aspectos clínicos perioperatórios de pacientes submetidos à cirurgia cardíaca. Braspen J. [Internet]. 2017. [citado 2021 mar 12];32(4):375-9. Disponível em: http://arquivos.braspen.org/journal/out-dez2017/13-Abreviscao-do-jejum.pdf 
22 Aguilar-Nascimento JE, Dock-Nascimento DB, Faria MSM, Maria EV, Yonamine F, Silva MR, et al. Ingestão pré-operatória de carboidratos diminui a ocorrência de sintomas gastrointestinais pós-operatórios em pacientes submetidos à colecistectomia. ABCD Arq Bras Cir Dig. 2007;20(2):77-80. doi: 10.1590/S010267202007000200002 .

23 Aguilar-Nascimento JE, Dock-Nascimento DB. Reducing preoperative fasting time: A trend based on evidence. World J Gastrointest Surg. 2010;2(3):57-60. doi: 10.4240\%2Fwjgs.v2.i3. 57.

24 Millard-Stafford MM, Wendland DM, O'Dea NK, Norman TL. Thirst and hydration status in everyday life. Nutr Rev. 2012;70(Suppl 2):14751. doi: 10.1111/j.1753-4887.2012.00527.x.

25 Bender MS, Jason SL, Franck LS, Lee KA. Theory of symptom management. In: Smith MJ, Liehr PR, editors. Middle range theory for nursing. $4^{\text {th }}$ ed. New York, NY: Springer Publishing Company; 2018. p. 147-78.

26 Armstrong LE, Muñoz CX, Armstrong EM. Distinguishing low and high water consumers: a paradigm of disease risk. Nutrients. 2020;12 (3):858. doi: 10.3390/nu12030858.

27 Dessote CAM, Furuya RK, Rodrigues HF, Rossi LA, Dantas RAS. Relation between stressors and hemodynamic instability in the postoperative period after cardiac surgery. Texto Contexto Enferm. 2018;27(3):e4530017. doi: 10.1590/0104-070720180004530017.

28 Garcia AKA, Fonseca LF, Lodi CR. O silêncio que permeia a sede perioperatória: um estudo de caso. In: Anais do $10^{\circ}$ Encontro Internacional de Produção Científica - EPCC. [Internet]; 2017 out 24-26. [citado $2021 \mathrm{abr}$ 12]. Disponível em: https://proceedings. science/epcc/papers/o-silencio-que-permeiaa-sede-perioperatoria \%3A-um-estudo-decaso?lang $=\mathrm{pt}$-br

29 Carlucci VDS, Rossi LA, Ficher AMFT, Ferreira E, Carvalho EC. Burn experiences from the patient's perspective. Rev Esc Enferm USP. 2007;41(1):21-8. doi: 10.1590/S008062342007000100003 .
30 Razera APR, Braga EM. The importance of communication during the postoperative recovery period. Rev Esc Enferm USP. 2011;45(3): 630-5. doi: 10.1590/S0080-62342011000300012.

31 Conchon MF, Fonseca LF. Efficacy of an ice popsicle on thirst management in the immediate postoperative period: a randomized clinical trial. J Perianesth Nurs. 2018;33(2):153-61. doi: 10.1016/j.jopan.2016.03.009.

32 Armstrong EL, Kavouras AS. Thirst and Drinking Paradigms: evolution from single factor effects to brainwide dynamic networks. Nutrients 2019;11(12):2864. doi: 10.3390/nu11122864.

33 Bichet DG. Vasopressin and the regulation of thirst. Ann Nutr Metab, 2018;72(Suppl 2):3-7. doi: $10.1159 / 000488233$.

34 Saker P, Farrell MJ, Egan GF, McKinley MJ, Denton DA. Influence of anterior midcingulate cortex on drinking behavior during thirst and following satiation. Proc Nat Acad Sci USA. 2018;15(4):786-91. doi: 10.1073/pnas. 171764 6115 .

35 Eccles R, Du-Plessis L, Dommels Y, Wilkinson JEE. Cold pleasure. Why we like ice drinks, ice-lollies and ice cream. Appetite. 2013;71: 357-60. doi: 10.1016/j.appet.2013.09.011.

Recebido em: 6 out. 2020

Aceito em: 1 set. 2021 